\title{
On the relationship between dynamic contrast-enhanced ultrasound parameters and the underlying vascular architecture extracted from acoustic angiography
}

Citation for published version (APA):

Panfilova, A., Shelton, S. E., Caresio, C., van Sloun, R. J. G., Molinari, F., Wijkstra, H., Dayton, P. A., \& Mischi, M. (2019). On the relationship between dynamic contrast-enhanced ultrasound parameters and the underlying vascular architecture extracted from acoustic angiography. Ultrasound in Medicine and Biology, 45(2), 539-548. https://doi.org/10.1016/j.ultrasmedbio.2018.08.018

Document license:

TAVERNE

DOI:

10.1016/j.ultrasmedbio.2018.08.018

Document status and date:

Published: 01/02/2019

Document Version:

Publisher's PDF, also known as Version of Record (includes final page, issue and volume numbers)

Please check the document version of this publication:

- A submitted manuscript is the version of the article upon submission and before peer-review. There can be important differences between the submitted version and the official published version of record. People interested in the research are advised to contact the author for the final version of the publication, or visit the DOI to the publisher's website.

- The final author version and the galley proof are versions of the publication after peer review.

- The final published version features the final layout of the paper including the volume, issue and page numbers.

Link to publication

\footnotetext{
General rights

- You may freely distribute the URL identifying the publication in the public portal. follow below link for the End User Agreement:

www.tue.nl/taverne

Take down policy

If you believe that this document breaches copyright please contact us at:

openaccess@tue.nl

providing details and we will investigate your claim.
}

Copyright and moral rights for the publications made accessible in the public portal are retained by the authors and/or other copyright owners and it is a condition of accessing publications that users recognise and abide by the legal requirements associated with these rights.

- Users may download and print one copy of any publication from the public portal for the purpose of private study or research.

- You may not further distribute the material or use it for any profit-making activity or commercial gain

If the publication is distributed under the terms of Article $25 \mathrm{fa}$ of the Dutch Copyright Act, indicated by the "Taverne" license above, please 


\title{
ON THE RELATIONSHIP BETWEEN DYNAMIC CONTRAST-ENHANCED ULTRASOUND PARAMETERS AND THE UNDERLYING VASCULAR ARCHITECTURE EXTRACTED FROM ACOUSTIC ANGIOGRAPHY
}

\author{
Anastasila Panfilova, ${ }^{*}$ Sarah E. Shelton,${ }^{\dagger}$ Cristina Caresio ${ }^{\dagger}$ Ruud J.G. van Sloun, ${ }^{*}$ \\ Filippo Molinari, ${ }^{\dagger}$ Hessel Wijkstra, ${ }^{*}, \S$ Paul A. Dayton,${ }^{\dagger}$ and Massimo Mischi* \\ * Department of Electrical Engineering, Technical University of Eindhoven, Eindhoven, The Netherlands; ${ }^{\dagger}$ Joint Department of \\ Biomedical Engineering, University of North Carolina at Chapel Hill and North Carolina State University, Chapel Hill, North \\ Carolina, USA; ${ }^{\ddagger}$ Politecnico di Torino, Torino, Italy; and ${ }^{\S}$ Urology Department, AMC University Hospital, Amsterdam, The \\ Netherlands
}

(Received 7 February 2018; revised 22 August 2018; in final from 27 August 2018)

\begin{abstract}
Dynamic contrast-enhanced ultrasound (DCE-US) has been proposed as a powerful tool for cancer diagnosis by estimation of perfusion and dispersion parameters reflecting angiogenic vascular changes. This work was aimed at identifying which vascular features are reflected by the estimated perfusion and dispersion parameters through comparison with acoustic angiography (AA). AA is a high-resolution technique that allows quantification of vascular morphology. Three-dimensional AA and 2-D DCE-US bolus acquisitions were used to monitor the growth of fibrosarcoma tumors in nine rats. AA-derived vascular properties were analyzed along with DCE-US perfusion and dispersion to investigate the differences between tumor and control and their evolution in time. AA-derived microvascular density and DCE-US perfusion exhibited good agreement, confirmed by their spatial distributions. No vascular feature was correlated with dispersion. Yet, dispersion provided better cancer classification than perfusion. We therefore hypothesize that dispersion characterizes vessels that are smaller than those visible with AA. (E-mail: A.P.Panfilova@tue.nl) (c) 2018 World Federation for Ultrasound in Medicine \& Biology. All rights reserved.
\end{abstract}

Key Words: Acoustic angiography, Dynamic contrast-enhanced ultrasound, Cancer, Dispersion, Perfusion, Ultrasound contrast agents.

\section{INTRODUCTION}

Malignant tissue differs from benign tissue in several aspects that can be used as markers to detect malignancy (Koumoutsakos et al. 2013). Of particular interest for cancer imaging are the altered vascular architecture and the consequent changes in blood supply. Angiogenic vessels grow to nourish the tumor and support its proliferation. These vessels have been found to be tortuous and to grow chaotically without the typical vessel hierarchy and with a high occurrence of arteriovenus shunts. Many of these properties can be recognized with contrast-enhanced ultrasound techniques, which have had promising results in distinguishing malignant from benign tissue (Brock et al. 2013; Gessner et al. 2013;

Address correspondence to: Anastasiia Panfilova, Department of Electrical Engineering, Technical University of Eindhoven, De Groene Loper 19, Eindhoven, The Netherlands. E-mail: A.P.Panfilova@tue.nl
Kuenen et al. 2011, 2013b; Mischi et al. 2012; Quaia 2011; Shelton et al. 2015).

Dynamic contrast-enhanced ultrasound (DCE-US) captures passage of the contrast agent through the vascular bed after its injection into the patient's bloodstream. Specifically, it registers the local evolution of gray-level intensity at each pixel, referred to as the time-intensity curve (TIC), which reflects the varying ultrasound contrast agent (UCA) concentrations. The recorded intensities are then converted into UCA concentration with a linearization function specific to the ultrasound scanner employed (Rognin et al. 2008), yielding an indicator dilution curve (IDC) for every pixel in the video. Various characteristics of IDCs have proven to be useful for distinguishing malignant from benign tissue (Mischi et al. 2012).

Several approaches have been adopted to extract information from IDCs derived from DCE-US bolus acquisitions. Some heuristic features of the IDCs, such 
as wash-in time and peak intensity, are related to cancer (Mischi et al. 2012; Zhao et al. 2010). Multiple other techniques employ IDC fitting with analytical models, such as the lognormal, gamma and local density random walk (LDRW) models (Strouthos et al. 2010). Functional parameters of the curves (e.g., area under the curve) are extracted and displayed in color maps, with the aim of obtaining a clearly distinguishable malignant region. All these approaches mainly attempt to quantify perfusion, which is motivated by the presence of ample arteries feeding the tumor, increased microvacular density (MVD) and the presence of arteriovenous shunts. Despite this, clinical evidence has indicated that cancerous lesions in the prostate can also be iso- or hypo-perfused (Brock et al. 2013). Indeed, it is known that tumor tissue has higher resistance to blood flow (Narang and Varia 2011). This induces a counterbalancing factor that complicates predictions on the level of blood supply within the tumor as compared with surrounding tissue (Cosgrove and Lassau 2010). Furthermore, the MVD inside the tumor can be strongly heterogeneous, creating highly perfused regions and hypoxic, avascular regions. Therefore, assessment of perfusion alone is insufficient for reliable cancer diagnostics. These findings have motivated the development of contrast ultrasound dispersion imaging (CUDI), a method that enables assessment of UCA dispersion, in addition to quantification of perfusion (Kuenen et al. 2011; Mischi et al. 2012).

CUDI is aimed at quantifying the UCA dispersion caused by the architecture of the vascular tree and complex multipath trajectories available for UCA transport. The main hypothesis underlying the method states that dispersion reflects structural vascular changes induced by angiogenesis. The first CUDI approach involved modeling of the IDCs in the time domain with an LDRW model and extraction of a dispersion-related parameter from the fitted model (Kuenen et al. 2011). An important complication associated with this approach was poor signal-to-noise ratio, hindering the fitting procedure and decreasing its reliability. This problem has been mitigated by spatiotemporal similarity analysis (Kuenen et al. 2013a; Mischi et al. 2012). In a promising implementation, this approach involves calculation of an average correlation coefficient measuring the similarity of a TIC at a pixel and its surrounding pixels (Kuenen et al. 2013b). A theoretical description of the problem within the framework of the LDRW model has revealed that the correlation coefficient between IDCs is monotonically related to the dispersion coefficient (Kuenen et al. 2013a). Moreover, this approach has been reported to have superior performance compared with perfusionrelated parameters in localizing prostate cancer in a clinical setting (Kuenen et al. 2013b). This method has been validated against cell differentiation reflected by the
Gleason score for prostate cancer (Schalk 2017). Another study found that regions of low dispersion correlated with those of high MVD, quantified by immunohistology (Saidov et al. 2016). However, in that study, detailed characterization of the vascular architecture (e.g., tortuosity and vessel size) was not available.

Acoustic angiography (AA) accurately characterizes the vascular architecture: It is a high-resolution technique capable of imaging individual microvessels (Gessner et al. 2013; Shelton et al. 2015). AA permits imaging of vessels at a high resolution of 100-200 $\mu \mathrm{m}$ at 2-cm depth with minimal signal from tissue. While transmitting ultrasonic waves at frequencies on the order of a few megahertz, close to the UCA bubble resonance frequency, it records the non-linear response of the contrast agents in a high-frequency range centered at $30 \mathrm{MHz}$. This technique makes it possible to quantify vessel density and morphology measures such as the sum-of-angles metric (SOAM) and distance metric (DM) (Rao et al. 2016; Shelton et al. 2015). These parameters have been reported to significantly differ between malignant and benign tissue (Gessner et al. 2013; Shelton et al. 2015). Thus, AA offers the opportunity to validate whether these features are reflected in DCE-US because of the different character of UCA perfusion and dispersion in these vessels.

The aim of this work was to determine whether DCE-US is able to characterize the underlying vascular architecture. We performed DCE-US and AA imaging of fibrosarcoma tumor-implanted and control regions in a longitudinal study of nine rats. AA and DCE-US acquisitions were performed every $3 \mathrm{~d}$, at four time points, starting the day the tumors could be palpated. An overall comparison of the vascular properties of the tumor and control regions was performed. Additionally, a longitudinal study of these properties was conducted with the aim of finding similar trends in features extracted with the two different techniques DCE-US and AA.

\section{METHODS}

\section{Rat models}

Fibrosarcoma tumor implantation was performed in rats according to a previously applied protocol (Streeter et al. 2011). The tumor models were established from propagated tumor tissue provided by the Dewhirst Lab at Duke University. Before surgery, the nine Fischer 344 rats were anesthetized with isoflurane; the left flank was then shaved and disinfected. An incision $(\sim 2 \mathrm{~mm})$ was made above the quadriceps muscle, and a sample of tumor tissue $\left(\sim 1 \mathrm{~mm}^{3}\right)$ was positioned under the skin. The incision was closed with one or two staples. This procedure was performed at three different time points 
with nine rats in total. Rats belonging to the same series were operated on the same day.

On day 8 after implantation, the first ultrasound acquisition was performed if the tumors were palpable. Otherwise, we waited 2 to 3 days for subsequent assessment. When the tumors were palpable, UCA was injected into the tail vein through a 24-gauge catheter while the animals were anesthetized with vaporized isoflurane in oxygen. DCE-US was performed on the tumor-bearing flank for assessment of perfusion and dispersion. The AA acquisition protocol immediately followed the DCE-US acquisition to minimize the amount of time each animal spent under anesthesia. The beginning of the DCE-US and AA acquisitions differed between the series, starting on days 8,11 and 13, respectively. For all but one animal, subsequent imaging acquisitions were performed with an interval of 3 days, resulting in four time points in total. In the one exception, we were not able to inject the contrast (for both modalities) in the tail vein and imaged only at the first and third time points. All experiments were performed at the University of North Carolina at Chapel Hill and were approved by the Institutional Animal Care and Use Committee at the University of North Carolina at Chapel Hill.

\section{Image acquisition}

DCE-US bolus injection protocol. A UCA bolus of $2 \times 10^{8}$ microbubbles was injected in the rats' tail vein. The contrast agent used in this study was made inhouse; it has a lipid shell and perfluorcarbon core, similar to Definity (Lantheus Medical Imaging, North Billerica, MA, USA). A 15L8-S probe was used with a Siemens Sequoia scanner in cadence pulse sequencing mode at an insonifying central frequency of $7 \mathrm{MHz}$. The acquired DCE-US recordings were stored in DICOM format.

AA continuous infusion protocol. A continuous infusion of microbubbles was administered using a syringe pump (PHD 2000, Harvard Apparatus) at the rate of $1.5 \times 10^{8}$ microbubbles per minute. AA imaging was performed with a dual-frequency single-element transducer transmitting at $4 \mathrm{MHz}$ and receiving at
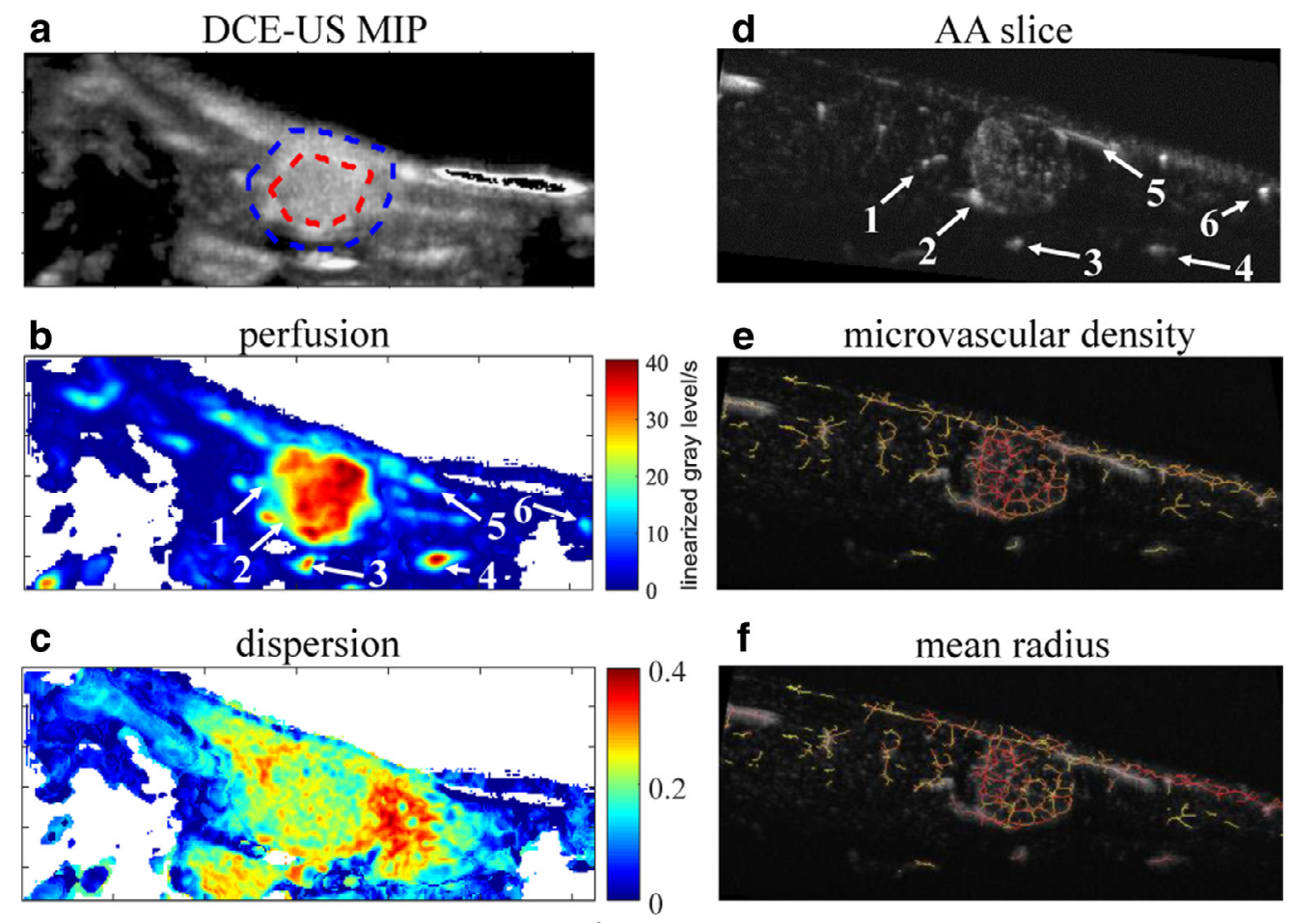

Fig. 1. Dynamic contrast-enhanced ultrasound and AA images of the same plane and maps of the extracted features. (a) MIP of the DCE-US video. The tumor is encircled by a red contour, and the region outside the blue contour belongs to the control, separated by a margin that was not included in the analysis. Regions with power below the threshold of $-22 \mathrm{~dB}$ of the maximum intensity are in black. (b, c) Perfusion (b) and dispersion (c) color maps, respectively. Regions with power below $-22 \mathrm{~dB}$ of the maximum intensity are in white. (d) Selected AA slice. (e, f) Vascular skeleton, colorcoded according to the values for microvascular density $€$ and mean radius (f), respectively (yellow indicates low values, and red indicates high values). The numbers in (b) and (d) are the numbers vessels identified in the perfusion maps, used as markers to locate the right plane in AA volumes. AA = acoustic angiography; DCE-US = dynamic contrast-enhanced ultrasound; MIP = maximum intensity projection 
around $30 \mathrm{MHz}$. The 3-D AA images were acquired plane by plane, with a step size of $100 \mu \mathrm{m}$.

\section{DCE-US bolus data processing}

Pre-processing. All bolus recordings were filtered with a Gaussian filter, as previously described in Mischi et al. (2012), using a kernel of $0.13 \mathrm{~mm}$ equal to 1.6 pixels. This value improved the signal-to-noise ratio at the cost of additional spatial correlation between TICs at neighboring pixels. The TIC power of every pixel was evaluated as the root mean square of the TIC after the baseline was removed. Regions with a level of TIC power below $-22 \mathrm{~dB}$ of the maximum TIC power over all images were excluded from further analysis (in black in Fig. 1a). This limited the effect of random noise on the parameters of interest (Kuenen et al. 2014). A characteristic of DCE-US is multiplicative noise: noise proportional in its power to the signal amplitude. By eliminating regions with low TIC power, we avoided erroneous parameter estimation from regions with low signal power where random noise dominates. After this, the intensity values of the remaining regions were linearized by inverting the logarithmic compression function implemented in the adopted scanner, yielding the IDCs.

Assessment of dispersion. An average correlation coefficient was calculated for every pixel between its own IDC and those of its surrounding pixels within a ring-shaped kernel (Mischi et al. 2012) with an inner radius of $0.6 \mathrm{~mm}$ and an outer radius of $2 \mathrm{~mm}$. The inner radius was chosen equal to the lateral resolution of the pre-processed bolus recordings at an $\sim 2$-cm depth as identified with local autocorrelation analysis. Details on the latter procedure can be found in Mischi et al. (2012). The lateral resolution was taken as a reference because it was worse than the axial resolution. The outer radius of the kernel was set equal to $2 \mathrm{~mm}$, a size that a tumor can usually reach without neovascularization (Folkman 1971). The time window over which the IDCs were correlated to each other was selected to maximize the area under the receiver operating characteristic curve for tumor classification, resulting in a value of $17 \mathrm{~s}$, as proposed in previous work (Panfilova et al. 2016). This is the only informative segment of the IDC (Fig. 2) because of early recirculation, as often observed in small animals (Stapleton et al. 2009). In this work, the beginning of the analyzed time window was set at $3 \mathrm{~s}$ before the appearance time, ensuring that the entire wash-in phase would be captured.

\section{Assessment of perfusion}

The wash-in-rate was adopted to assess perfusion and computed as the slope of a line fitted to the IDC in

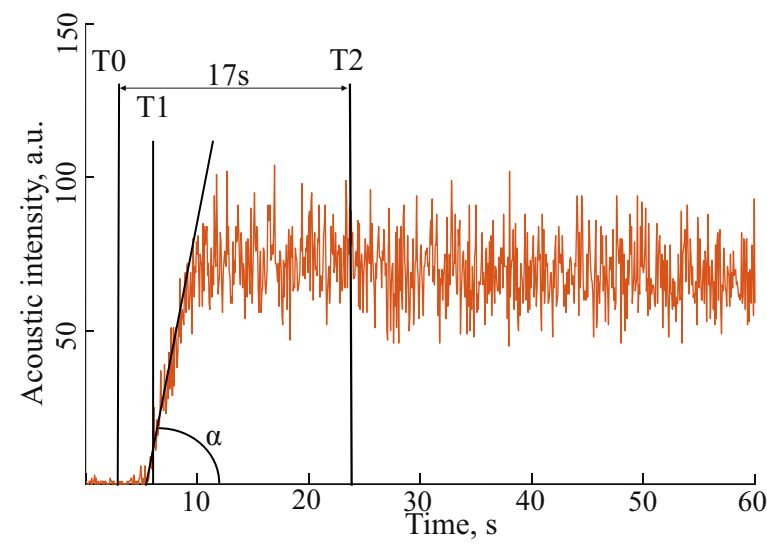

Fig. 2. Typical pre-processed indicator dilution curve. T1 is the time of appearance. T0 is $3 \mathrm{~s}$ before $\mathrm{T} 1$. The interval from $\mathrm{T} 0$ to $\mathrm{T} 2$ is the interval of the indicator dilution curve used for dispersion analysis. The tangent of the angle $\alpha$ of the line fitted to the indicator dilution curve in the $2 \mathrm{~s}$ after T1 is the wash-in rate.

the 2-s interval after appearance time, as illustrated in Figure 2. The value of $2 \mathrm{~s}$ was chosen to reflect the rise in UCA concentration in the initial part of the IDCs in all acquired clips.

\section{AA data processing}

Acoustic angiography volumes were interpolated to reduce the inter-plane distance to $50 \mu \mathrm{m}$ and make the pixels isotropic. Visible vessels were manually segmented and characterized in terms of vessel dimensions: vessel length (VL) and mean radius (MR). VL was computed as the length of the vessel segment identified between successive branching points, and MR was computed as the mean radius of this vessel segment along its length. Vessel tortuosity was assessed with the DM and the SOAM (Bullitt et al. 2003). The DM was computed as the ratio of vessel length to Eucledian distance between its beginning and end. The SOAM was calculated as the sum of angles between successive points on the vessel centerline divided by VL, using the same formula as described in Bullitt et al. (2003), but excluding the torsional angle. In addition to these individual vessel properties, MVD was calculated as a global characteristic of the tumor at a given time point, defined as the number of visible vessel segments divided by tumor volume. Volume vascular density (VVD) was computed with a moving 3-D isotropic kernel in the central slice of the tumor ( $\sim \mathrm{mm}$ thick). Otsu's method (Vala and Baxi 2013) was used to select a threshold to separate noise from vessel signal within the central slices; the percentage of pixels with vessel signal from the overall number of pixels in the 3-D kernel was calculated. 


\section{Statistical analysis}

The DCE-US parameters were spatially downsampled by a factor of 7 in both directions, equal to the resolution of the pre-processed images. This was performed to exclude spatial correlation and prepare the data for the statistical tests that require sample independence.

Comparison between tumor and control regions. Dispersion and perfusion values were divided into two groups. The tumor group was composed of manually selected tumor regions (inside the red contour, Fig. 1a) from all rats at all time points binned together. The control group was taken from pixels outside the tumor contour, dilated by $\sim 1 \mathrm{~mm}$ (in blue, Fig. 1a). The region between the red and blue contours was excluded from analysis to make our pixel classification robust to inaccurate tumor delineation by DCE-US contouring. AA parameters were extracted in a similar fashion: vessels were taken from within the tumor region and outside it in the same flank (Fig. 3). We eliminated from analysis those vessel segments on the border of the selected contour whose tumor or control origin was unknown.

An Anderson-Darling goodness-of-fit hypothesis test was performed on all parameter distributions to check for data normality. Because all the distributions were identified as non-Gaussian, a Mann-Whitney non-parametric test was performed to establish the significance ( $p$ value) of the difference between tumor and control. No additional subsampling or upsampling was performed to balance the control and tumor data sets because the Mann-Whitney test can be applied to data sets with distributions of different size (Mann and Whitney 1947).

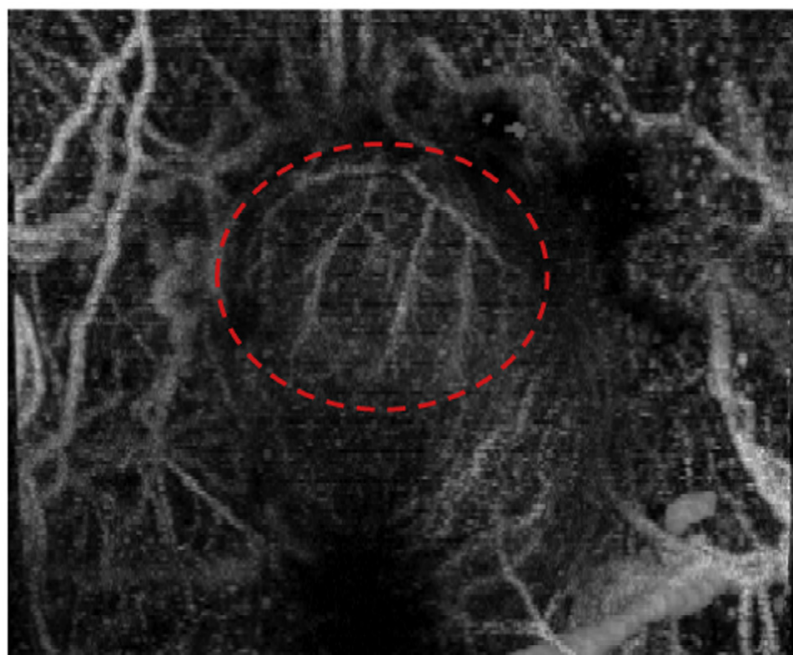

Fig. 3. Acoustic angiography maximum intensity projection. The tumor region is indicated by the red contour, surrounded by the control region.
Cohen's $d$ was used as a measure of the "effect size" (Sullivan and Feinn 2012) the tumor has on the underlying vasculature, calculated as the difference between the means of two distributions divided by the standard deviation of the control. The values of Cohen's $d$ allow classification of the difference between two distributions into four categories: small, medium, large and very large for values of $0.2,0.5,0.8$ and 1.3 , respectively.

\section{Longitudinal study of tumor and control}

A longitudinal study of tumor evolution was performed with the Kruskall-Wallis (Kruskal and Wallis 1952) test followed by Tukey's post hoc test (implemented in the function "multcompare" in MATLAB), evaluating the differences among the distributions of dispersion and perfusion, and vascular features of tumor and control regions at four time points. The Kruskall-Wallis test does not require equal sample sizes, which is an advantage considering that our data set is unbalanced and incomplete: Data are missing for one tumor region at two time points, as well as for control regions at several time points for the large tumors. Moreover, the number of visible vessels differed for every image acquisition. For all rats, all parameter values were binned together according to the time point of the acquisition.

The statistical test calculation is influenced by the number of observations and can result in different outcomes for different sample sizes (Kruskal and Wallis 1952). Because the number of pixels provided more samples for dispersion and perfusion compared with the number of vessels extracted with AA, these pixels were randomly subsampled to yield the same number of samples as vessels per each representative data set of tumor and control regions at each time point. The only parameter that remained different in terms of group size was MVD because it is a global parameter that characterizes the entire tumor and control at a specific time point.

After the post hoc tests were performed, the Pearson correlation coefficient was computed between the medians of the parameters showing similar longitudinal trends.

Mapping of vascular properties on the bolus acquisition plane. During the DCE-US bolus acquisitions the operator always tried to image the largest cross section of the tumor and maintain the same orientation of the probe as used for AA. However, it was noted that these precautions were not sufficient to reliably identify the DCE-US plane with AA: even a movement on the order of $\sim 1 \mathrm{~mm}$ alters the imaged vascular pattern of a tumor. The perfusion maps were found to highlight larger vessels, clearly visible in the AA (Fig. 2b, d). These vessels were used as markers to locate the bolus recording plane in the AA volume. For this, a dedicated 
tool was developed, allowing free scrolling through the AA volume planes and changes in their orientation.

The plane was selected by visual inspection, choosing an image containing as many of the vessel markers present in the perfusion maps as possible. A slice in the AA volume $\sim 1 \mathrm{~mm}$ thick was selected, and an extension of the skeletonization algorithm described in Meiburger et al. (2016) was applied to extract MVD (Fig. 2e), MR (Fig. 2f), VL, and SOAM. This slice thickness was chosen to be on the order of the elevational resolution in the bolus recordings and sufficiently large to register vessel segments. This allowed a qualitative comparison of the spatial distribution of the vascular features with those of dispersion and perfusion in the same plane.

All image processing and statistical analysis were performed with MATLAB software (The MathWorks, Natick, MA, USA).

\section{RESULTS}

\section{Statistical analysis}

Comparison between tumor and control. For all extracted parameters, tumor and control regions had significantly different distributions $(p<0.001)$. However, the magnitude of the differences, expressed as Cohen's $d$, spans a wide range (Fig. 4), with a marginal effect size for the DM (Fig. 4c) and small to very large differences for the remaining parameters.

Longitudinal study of tumor and control. Since the DM showed almost no difference between tumor and control, it was excluded from the longitudinal analysis.
Boxplots with all parameter values binned according to the time points are shown in Fig. 5, while Fig. 6 illustrates the results of Tukey's post hoc test, color-coded according to the significance level of the intra-distribution differences.

The dispersion median is relatively constant in time for both tumor and control, showing a significant difference for control and tumor distributions (Figs. 5a and 6a). Tumor perfusion is significantly different from that of the control at all time points (Figs. $5 \mathrm{~b}$ and $6 \mathrm{~b}$ ), peaking for the tumors at the second time point. Interestingly, the longitudinal trend of the control's perfusion seems to mimic the tumor's trend in time, although at a smaller magnitude and not identified as significant with the post hoc test.

The VVD is stably higher for tumor, whereas MVD seems to follow a trend similar to that of perfusion, peaking for tumors at the second time point. However, the results of the MVD post hoc test are difficult to compare with other results because the number of samples differed: Only one value of MVD per time point was available, whereas the other parameters were subsampled with respect to the number of segmented vessels in the AA volume at a given time point.

The post hoc results, illustrated by color maps in Figure 6, are comparable for dispersion, VVD, VL and SOAM. However, there was no significant correlation between the medians of the dispersion levels and the aforementioned AA parameters. As for perfusion, the mean perfusion in tumors and their MVD were significantly correlated, with a coefficient of $0.572(p<0.001)$, and inclusion of both control and tumor values resulted in a Pearson correlation coefficient of $0.67(p<0.001)$. a $\quad \mathrm{d}=1.68$
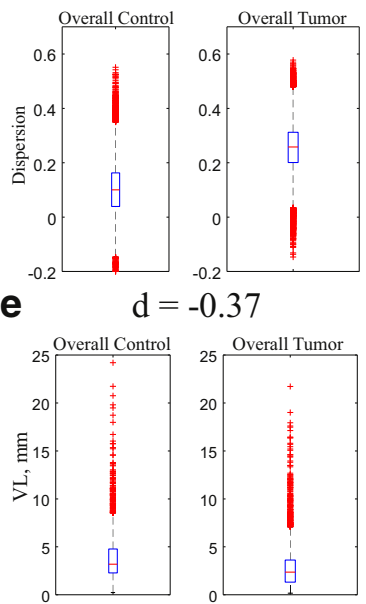

b

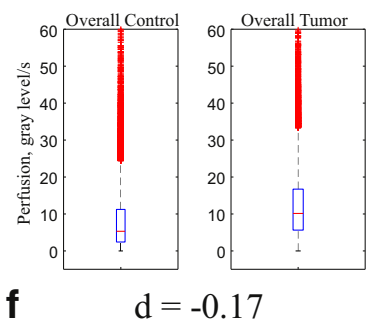

f

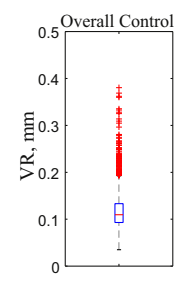

C

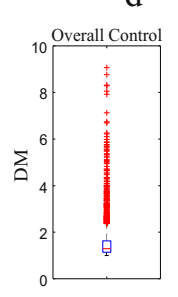

$\mathrm{d}=0.06$

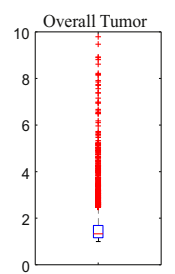

g
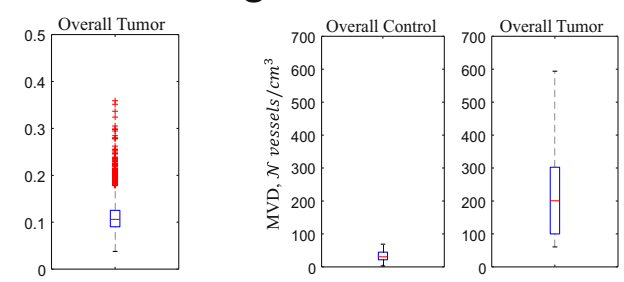

d

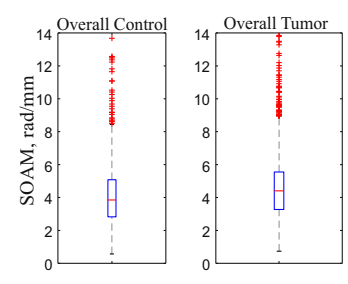

h

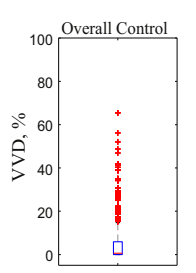

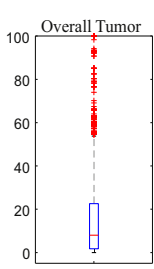

Fig. 4. Boxplots of tumor and control parameters binned together from all time points. (a) Dispersion. (b) Perfusion. (c) Distance metric, DM. (d) Sum-of-angles metric, SOAM. (e) Vessel length, VL. (f) Vessel radius, VR. (g) Microvascular density, MVD. (h) Volume vascular density, VVD. Cohen's $d$ is indicated above the plots. 
a

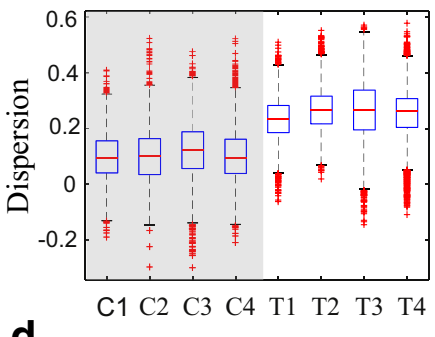

d

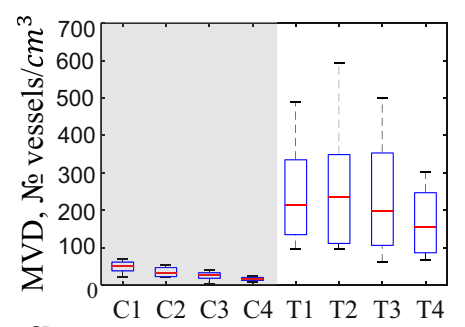

g

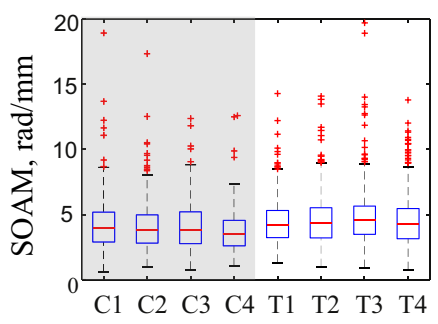

b

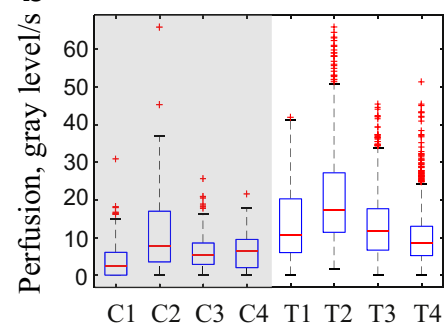

e

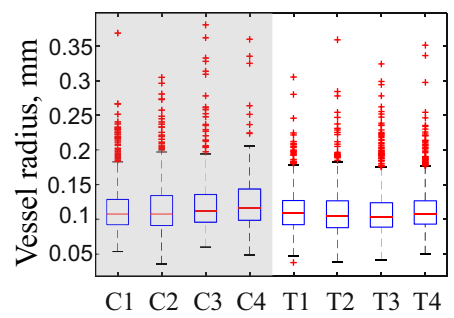

C

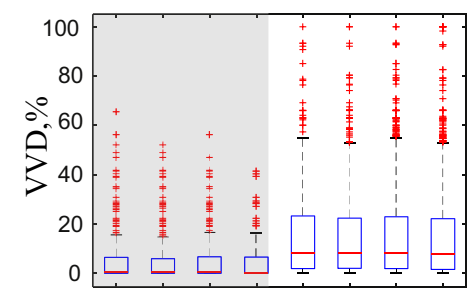

$\begin{array}{llllllll}\mathrm{C} 1 & \mathrm{C} 2 & \mathrm{C} 3 & \mathrm{C} 4 & \mathrm{~T} 1 & \mathrm{~T} 2 & \mathrm{~T} 3 & \mathrm{~T} 4\end{array}$

\section{f}

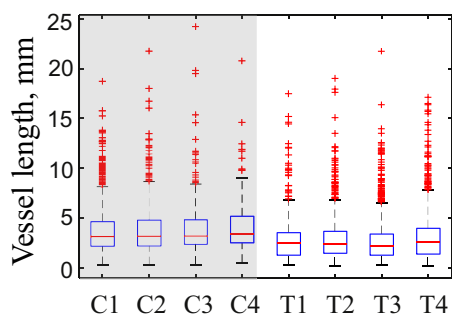

Fig. 5. Boxplots of tumor (T1, T2, T3, T4) and control (C1, C2, C3, C4) parameters, binned together at different time points. (a) Dispersion. (b) Perfusion. (c) Volume vascular density, VVD. (d) Microvascular density, MVD. (e) Vessel radius, VR. (f) Vessel length, VL. (g) Sum-of-angles metric (SOAM).

Mapping of vascular properties on the bolus acquisition plane

The spatial parametric maps of the AA skeleton confirmed our observation that there is a correlation between regions of high perfusion and elevated MVD (Fig. 1b, f). No spatial correspondence was found between dispersion and the other AA-derived parameters.

\section{DISCUSSION}

Dispersion differed greatly (Cohen's $d=1.68$ ) between tumor and control regions, exhibiting stable performance at tumor detection as it developed. Perfusion had a lower discrimination power than dispersion; it was high for younger tumors, peaking at time point 2, and decreased with tumor growth. Interestingly, the perfusion level in the control around the tumor was also elevated (Fig. 5 b) and followed a trend similar to that of the tumor itself. This may reflect that the overall perfusion of tissue around the tumor was increased and influenced by the tumor. This effect has been observed for the SOAM, which exhibits intermediate values between those of the tumor and control regions in tissue adjacent to the tumor (Rao et al. 2016). Moreover, for the fibrosarcoma mode, it has been found that the vascular source is often located in the periphery of the tumor (Ponce et al. 2007; Tozer et al. 1990; Viglianti et al. 2004).

Dispersion of the control remains stable over time, indicating that dispersion-related changes occur mainly within the tumor itself and not in the surroundings. The spatial perfusion and dispersion maps are complementary, exhibiting different patterns of highlighted regions (Fig. 1b, c). Perfusion highlights large vessels, as well as regions with high MVD.

The SOAM indicates that the tumor has more tortuous vessels and follows a trend similar to that of dispersion (Fig. 5a, g), with comparable results for the post hoc test (Fig. 6a, g). Nevertheless, the effect size difference, as indicated by Cohen's $d$, was much lower for the SOAM than for dispersion. In general, the control regions in this experiment exhibits a higher tortuosity than we previously observed for these rats, expressed by the DM in Shelton et al. (2015). A direct comparison of the SOAM in this work with that in Shelton et al. (2015) is not available because the calculation of the SOAM has been adjusted since that time. The unusually high tortuosity of the 


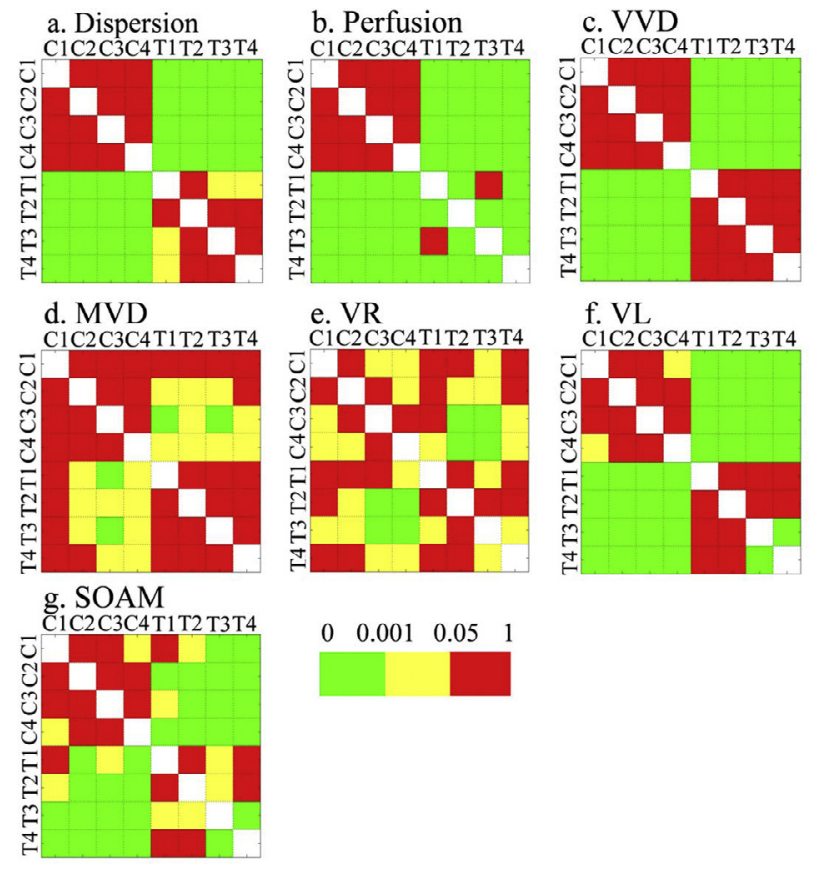

Fig. 6. Results of Tukey's post hoc test performed on tumor and control parameters at four time points (indicated by $\mathrm{T} 1$, $\mathrm{T} 2, \mathrm{~T} 3$ and $\mathrm{T} 4$ and by $\mathrm{C} 1, \mathrm{C} 2, \mathrm{C} 3$ and $\mathrm{C} 4$, respectively). The colors of the rows indicate whether the distribution significantly differs from the others, with green and yellow representing different significance levels. (a) Dispersion. (b) Perfusion. (c) Volume vascular density, VVD. (d) Microvascular density, MVD. (e) Vessel radius,VR. (f) Vessel length, VL. (g) Sum-ofangles metric, SOAM.

control may be caused by the presence of the bowel region in some of the AA images, which was excluded from analysis in earlier studies and may have elevated tortuosity. Previous data also indicated that the SOAM exhibited an intermediate level of tortuosity in tissue up to $1 \mathrm{~cm}$ away from tumor, with a mean tortuosity between that of tumors and non-tumor-bearing animals (Rao et al. 2016). The discrimination power of SOAM in our data set increases for smaller vessels (Cohen's $d=0.14$ for vessels with a radius $>0.11 \mathrm{~mm}, 0.28$ with an intermediate radius and 0.43 with a radius $<0.09 \mathrm{~mm}$ ). Therefore, its relationship to the extracted DCE-US features cannot be fully appreciated because of the finite resolution of AA. Similarly, a previous study reported that the difference in MVD between tumor and control increases for smaller vessels (Sedelaar et al. 2001). Therefore, it may be that the SOAM, MVD and other metrics extracted in this study are related to dispersion; however, it is mainly the properties of the smaller vessels that have a significant influence on it. Supporting this hypothesis is the former observation that regions with increased MVD correspond to those with low dispersion (Saidov et al. 2016), as derived from immunohistology. The immunohistology-derived MVD was based on evaluation of tomato lectin binding to endothelial cells and therefore characterizes the presence of vessels of all sizes.

Spots of increased vascular density or large vessels were detected with perfusion color maps. The correlation between median perfusion level and MVD is the only significant inter-parameter agreement found in this work.

The Kruskall-Wallis test is ideally constructed for a study design in which subjects are randomly assigned to different groups so that each subject appears in one group only (Kruskal and Wallis 1952). Moreover, the subjects within the group must be independent. We realize that these assumptions are not strictly valid in this study because we observed tumor evolution in the same rats over time and because vessels selected from the same rat are, strictly speaking, not independent. However, we do not expect these limitations to be crucial in deriving a meaningful conclusion about the significant trends in time.

Imaging initialization differed among three series of experiments, starting with days 8, 11 and 13 after tumor implantation, as explained previously. We think that combining all rats according to the number of the acquisition is justified because the imaging was initialized according to the same strategy: when the tumors became palpable. However, because we waited 2-3 days for subsequent assessment if tumors were not pulpable on day 8 , in future work it may be beneficial to assess the tumors every day or to evaluate all tumors in a single cohort. This would ensure that the development of the imaged tumors is more consistent.

It is often observed that the wash-out phase is masked by recirculation in small animals. Stapleton et al. (2009) reported that for a range of administered UCA doses, the wash-out phase is more prominent in mice. Different UCA doses should therefore be investigated in our future work because a prominent wash-out phase, in our experience, enhances the performance of CUDI (Kuenen et al. 2013b). A clear wash-out would also allow evaluation of the wash-out as a complementary perfusion parameter.

An important limitation of this study is the 2-D character of the extracted parameters dispersion and perfusion. The results of the post hoc tests, therefore, must still be taken with caution because these tests were performed for 3-D vascular features evaluated in the whole tumor volume and 2-D dispersion and perfusion that leave us blind to out-of-plane information and restrict us to the central tumor slice, which is not always representative of the whole tumor (Streeter et al. 2011). We mitigated this limitation by performing an additional spatial comparison of the parameter maps in the same plane, matched with the help of large vessels identified in the perfusion maps. The agreement between perfusion and MVD, observed in the longitudinal trends, was also 
identified in the spatial distribution of these parameters in the same plane, increasing confidence in the finding that perfusion and MVD are correlated.

An improved study design should either include 3-D DCE-US (Schalk et al. 2015), yielding more accurate overall tumor characteristics, or a registration procedure, allowing fixation of the orientation of the probes and identification of the location of the DCE-US plane within the AA volume. The finding that perfusion highlights large vessels can be used to further improve registration.

The lack of parameters correlated with dispersion may pinpoint to the limitation of AA as a validation method for CUDI: Although enabling very high resolution ultrasound imaging, it may not be sufficient to determine which vascular properties substantially influence dispersion because dispersion may be defined mainly by properties of subresolution vessels. In this respect, it is possible to direct our attention to superlocalization methods that overcome the limit of diffraction: They are able to track single bubbles and determine their exact positions by finding the centers of their point spread functions (Cox and Beard 2015; Errico et al. 2015). Another possible reason for the lack of vascular parameters that correlate with dispersion is that the adopted dispersion parameter is in fact related to both dispersion and flow velocity (Kuenen et al. 2013a). Different vascular parameters may contribute to the separate terms of dispersion and flow velocity, whereas we assessed their combination. In this regard, it would also be of interest to apply another analysis to the DCE-US bolus recordings that allows us to separate dispersion and velocity contributions (van Sloun et al. 2017).

\section{CONCLUSIONS}

In this work, dispersion was found to have superior performance in tumor classification compared with perfusion, as previously found for prostate cancer (Kuenen et al. 2013a, 2013b; Mischi et al. 2012). Perfusion color maps highlight large vessels and regions of elevated MVD. The vascular factors that determine the dispersion level remain to be found, as does the role of vessels with a diameter $<100-200 \mu \mathrm{m}$ in defining perfusion levels.

Acknowledgments-This work was supported by European Research Council Starting Grant 280209 and the Impulse2 program within TU/e and Philips. This work was also supported by Grants R01CA170665, R43CA165621 and R01CA189479 from the National Institutes of Health.

Conflict of interest disclosure-P.A.D. declares that he is an inventor on a patent enabling the acoustic angiography technology and a cofounder of SonoVol, Inc., a company that has licensed this technology.

\section{REFERENCES}

Brock M, Eggert T, Rein JP, Roghmann F, Braun K, Löppenberg B, Sommerer F, Noldus J, Bodman C. Multiparametric ultrasound of the prostate: Adding contrast enhanced ultrasound to real-time elastography to detect histopathologically confirmed cancer. J Urol 2013;189:93-98.

Bullitt E, Gerig G, Pizer SM, Lin W, Aylward SR. Measuring tortuosity of the intracerebral vasculature from MRA images. IEEE Trans Med Imaging 2003;22:1163-1171.

Cosgrove D, Lassau N. Imaging of perfusion using ultrasound. Eur J Nucl Med Mol Imaging 2010;37:65-85.

Cox B, Beard P. Super-resolution ultrasound. Nature 2015;527:451452.

Errico C, Pierre J, Pezet S, Desailly Y, Lenkei Z, Couture O, Tanter M. Ultrafast ultrasound localization microscopy for deep super-resolution vascular imaging. Nature 2015;527:499-502.

Folkman J. Tumor angiogenesis: Theraputic implications. N Engl J Med 1971;285:1182-1186.

Gessner R, Frederick C, Foster F, Dayton P. Acoustic angiography: A new imaging modality for asessing microvascular architecture. Int J Biomed Imaging 2013;2013 936593.

Koumoutsakos P, Pivkin I, Milde F. The fluid mechanic of cancer and its therapy. Annu Rev Fluid Mech 2013;45:325-355.

Kruskall WH, Wallis WA. Use of ranks in one-criterion variance analysis. J Am Stat Assoc 1952;47:583-621.

Kuenen MPJ, Mischi M, Wijkstra H. Contrast-ultrasound diffusion imaging for localization of prostate cancer. IEEE Trans Med Imaging 2011;30:1493-1502.

Kuenen M, Saidov T, Wijkstra H, Mischi M. Contrast-ultrasound dispersion imaging for prostate cancer localization by improved spatiotemporal similarity analysis. Ultrasound Med Biol 2013a;39:1631-1641.

Kuenen M, Saidov T, Wijkstra H, Mischi M. Spatiotemporal correlation of ultrasound-contrast-agent dilution curves for angiogenesis localization by dispersion imaging. IEEE Trans Ultrason Ferroelectr Freq Control 2013b;60:2665-2669.

Kuenen MPJ, Herold IHF, Korsten HHM, de la Rosette JJMCH, Wijkstra H. Maximum-likelihood estimation for indicator dilution analysis. IEEE Trans Biomed Eng 2014;61:821-831.

Mann HB, Whitney D. On a test of whether one of two random variables is stochastically larger than the other. Ann Math Stat 1947;18:50-60.

Meiburger KM, Nam S, Chung E, Suggs LJ, Emelianov SY, Molinari F. Skeletonization algorithm-based blood vessel quantification using in vivo 3D photoacoustic imaging. Phys Med Biol 2016;61:7994-8009.

Mischi M, Kuenen MPJ, Wijkstra H. Angiogenesis imaging by spatiotemporal analysis of ultrasound-contrast-agent dispersion kinetics. IEEE Trans Ultrason Ferroelectr Freq Control 2012;59:621-629.

Narang AS, Varia S. Role of tumor vascular architecture in drug delivery. Adv Drug Delivery Rev 2011;63:640-658.

Panfilova A, Shelton S, Sloun R, Demi L, Wijkstra H, Dayton P, Mischi M. Does contrast ultrasound dispersion imaging reveal changes in tortuosity? A comparison with acoustic angiography. Proc IEEE Int Ultrason Symp 2016;1-4.

Ponce A, Viglianti B, Yu D, Yarmolenko P, Michelich C, Woo J, Bally M, Dewhirst M. Magnetic resonance imaging of temperature-sensitive liposome release: Drug dose painting and antitumor effects. J Natl Cancer Inst 2007;99:53-63.

Quaia E. Assessment of tissue perfusion by contrast-enhanced ultrasound. Eur Radiol 2011;21:604-615.

Rao S, Shelton S, Dayton P. The 'fingerprint' of cancer extends beyond solid tumor boundaries: Assessment with a novel ultrasound imaging approach. IEEE Trans Biomed Eng 2016;63:1082-1086.

Rognin NG, Frinking P, Costa M, Arditi M. In-vivo perfusion quantification by contrast ultrasound: Validation of the use of linearized video data vs. raw RF data. Proc IEEE Int Ultrason Symp 2008;1690-1693.

Saidov T, Heneweer C, Keunen M, Broich-Oppert J, Wijkstra H, Rosette J, Mischi M. Fractal dimension of tumor microvasculature by DCE-US: Preliminary study in mice. Ultrasound Med Biol 2016;42:2852-2863. 
Schalk S. Towards 3D prostate cancer localization by contrast ultrasound dispersion imaging. Ph.D. thesis. : Eindhoven University of Technology; 2017.

Schalk SG, Demi L, Smeenge M, Millis DM, Wallace K, de la Rosette J, Wijkstra H, Mischi M. 4D spatiotemporal analysis of ultrasound contrast agent dispersion for prostate cancer localization: A feasibility study. IEEE Trans Ultrason Ferroelectr Freq Control 2015;62:839-851.

Sedelaar JM, Leenders G, Hulsbergen-van de Kaa C, Poel H, Laak J, Debruyne F, Wijkstra H, Rosette J. Microvessel density: Correlation between contrast ultrasonography and histology of prostate cancer. Eur Urol 2001;40:285-293.

Shelton S, Lee Y, Lee M, Cherin E, Foster F, Aylward S, Dayton P. Quantification of microvascular tortuosity during tumor evolution utilizing acoustic angiography. Ultrasound Med Biol 2015;41:1869-1904.

Stapleton S, Goodman H, Yu-Qing Z, Cherin E, Henkelman M, Burns PN, Foster FS. Acoustic and kinetic behavior of Definity in mice exposed to high frequency ultrasound. Ultrasound Med Biol 2009;35:296-307.

Streeter JE, Gessner RC, Tsuruta J, Feingold S, Dayton PA. Assessment of molecular imaging of angiogenesis with three-dimensional ultrasonography. Mol Imaging 2011;10:460-468.
Strouthos C, Lampaskis M, Sboros V, McNeilly A, Averkiou M. Indicator dilution models for the quantification of microvascular blood flow with bolus administration of ultrasound contrast agents. IEEE Trans Ultrason Ferroelectr Freq Control 2010;57:1296-1310.

Sullivan MG, Feinn R. Using effect size-Or why the $p$-value is not enough. J Grad Med Educ 2012;4:279-282.

Tozer GM, Lewis S, Michalowski A, Aber V. The relationship between regional variations in blood flow and histology in a transplanted rat fibrosarcoma. Br J Cancer 1990;61:250-257.

Vala HJ, Baxi A. A review on Otsu image segmentation algorithm. Int J Adv Res Comput Sci Softw Eng 2013;2:387-389.

van Sloun RJ, Demi L, Postema AW, de la Rosette JJ, Wijkstra H, Mischi M. Ultrasound-contrast-agent dispersion and velocity imaging for prostate cancer localization. Med Image Anal 2017;35:610-619.

Viglianti B, Abraham S, Michelich C, Yarmolenko P, MacFall J, Bally M, Dewhirst M. In vivo monitoring of tissue pharmacokinetics of liposome/drug using MRI: Illustration of targeted delivery. Magnet Reson Med 2004;51:1153-1162.

Zhao H, Xu R, Ouyang Q, Chen L, Dong B, Huihua Y. Contrastenhanced ultrasound is helpful in the differentiation of malignant and benign breast lesions. Eur J Radiol 2010;73:288-293. 